\title{
Les pédagogies alternatives en France aujourd'hui : essai de cartographie et de définition
}

\section{Sylvain Wagnon}

\section{(2) OpenEdition}

\section{Journals}

Édition électronique

URL : http://journals.openedition.org/trema/4174

DOI : $10.4000 /$ trema.4174

ISSN : 2107-0997

Éditeur

Faculté d'Éducation de l'université de Montpellier

Édition imprimée

Date de publication : 1 novembre 2018

ISSN : 1167-315X

Référence électronique

Sylvain Wagnon, «Les pédagogies alternatives en France aujourd'hui : essai de cartographie et de définition », Tréma [En ligne], 50 | 2018, mis en ligne le 01 novembre 2018, consulté le 10 décembre 2020. URL : http://journals.openedition.org/trema/4174 ; DOI : https://doi.org/10.4000/trema.4174

Ce document a été généré automatiquement le 10 décembre 2020.

Trema 


\title{
Les pédagogies alternatives en France aujourd'hui : essai de cartographie et de définition
}

\author{
Sylvain Wagnon
}

\section{Introduction}

1 Indéniablement, les pédagogies alternatives suscitent intérêt et interrogation. Cet essor, réel même si quantitativement très limité, doit être observé, analysé, étudié dans ses modalités et dans les défis qu'il pose au système éducatif dans son ensemble.

2 Pédagogies alternatives, le terme annonce une autre façon d'éduquer, d'enseigner, de comprendre les apprentissages, les relations humaines, pour repenser notre société. L'écart est encore abyssal entre le nombre d'enfants scolarisés dans des structures alternatives, quelques dizaines de milliers et «l'attirance» pour une autre façon d'éduquer et une volonté de changer l'école dite traditionnelle. Mais comment définir ces pédagogies alternatives et comprendre leurs finalités éducatives?

3 Nous avons choisi d'étudier les propositions et les discours des acteurs de ces pédagogies, dans leur diversité et de mettre en avant les principes fédérateurs de ces pédagogies sans toutefois omettre les oppositions et les conflits entre ces mouvements.

$4 \quad$ Nous nous devions aussi de réfléchir aux finalités éducatives et nécessairement politiques qu'elles proposent. Ces écoles sont-elles, comme la plupart des acteurs de ces écoles le soulignent, l'illustration de l'essor d'une autre vision de notre société avec des rapports humains plus bienveillants et plus fraternels pour le bien-être des enfants ou, de façon non assumée, une volonté de repli sur soi?

5 Les militants de ces écoles ne doivent pas être choqués par cette dernière interrogation. C'est une tension perceptible par toutes les écoles alternatives depuis celles des courants d'éducation nouvelle du début du XXe siècle. La question de la mixité sociale des écoles 
alternatives doit être posée car c'est un élément de leur possible essaimage. Si ces écoles veulent réellement être des alternatives, elles se doivent de réfléchir à cet état de fait.

Délimiter la galaxie des pédagogies alternatives, c'est à la fois prendre en compte une multitude de "chapelles ", d'îlots éducatifs qui possèdent des liens plus ou moins étroits entre eux. Quel point commun entre une école « traditionnaliste » religieuse et une école Montessori, entre une école Freinet et le homeschooling? Analyser cette nébuleuse c'est aussi tenter d'établir les principes fédérateurs de ces mouvements sans sous-estimer les oppositions entre les acteurs de ces différents courants. Comprendre les pédagogies alternatives c'est aussi souligner le rôle effectif des initiateurs, des soutiens et des ramifications idéologiques et financières. Certains sites médiatiques comme le Printemps de l'éducation ou le Festival de l'école de la vie tentent d'accueillir et de fédérer ces groupes hétérogènes, ils sont des moyens d'observer les interactions entre des personnalités, des idéologies et des courants de pensée.

7 Composée actuellement principalement d'établissements privés hors contrat, la question des liens avec l'institution scolaire est posée. En étudiant les discours des différents groupes, on se rend bien compte que les «attaques" contre l'enseignement public ne sont pas toutes de même nature. Certains mouvements prônent des stratégies de contournement ou de concurrence. S'agit-il pour eux, tout en appelant au dialogue entre privé et public, d'être en concurrence avec l'enseignement public et d'apparaître comme des poissons-pilotes de la libéralisation et de la privatisation de l'éducation? S'agit-il d'une opposition de principe à l'enseignement public ou d'une résistance plus conjoncturelle ? Là aussi, il convient de rappeler que les courants d'éducation nouvelle du début du XXe siècle, dans leurs oppositions au système éducatif dit classique se sont en grande partie développés à la marge. Mais certains acteurs de ces pédagogies, tout en critiquant l'institution, ont la volonté de transformer l'enseignement public, de l'intérieur comme c'est le cas pour le mouvement Freinet et peut-être en train de s'esquisser avec le courant Montessori'. Il ne s'agit aucunement, pour le mouvement Freinet, d'une volonté de détruire l'enseignement public même si parfois certaines critiques convergent avec celles de groupes qui clairement veulent la fin d'un modèle public d'éducation.

8 Une cartographie nous est apparue nécessaire pour expliciter la variété de la nébuleuse des courants dits des pédagogies alternatives mais aussi pour analyser leurs caractéristiques, les liens entre les différents acteurs de cette mouvance. Quels sont les contours de cette nébuleuse pour reprendre le terme de Christian Topalov (1999) ? Quels sont les invariants, les principes directeurs, les pratiques et les finalités communes de ces écoles et de ces « pédagogies »? Quelles sont leurs aspirations, leur volonté de changer le paradigme scolaire? En étudiant les écrits de leurs militants, les liens entre les $\mathrm{s}$ nombreux «conférenciers » et diffuseurs de ces courants nous avons mis en lumière les ramifications implicites et explicites entre ces différents "archipels» dans l'espace français. Ce travail n'est qu'une première étape d'une cartographie plus globale et internationale.

9 Ainsi tous ces questionnements nous amènent-ils à nous interroger sur l'essor actuel des pédagogies alternatives comme révélateur des mutations de l'école et des relations entre les parents et l'institution scolaire. En effet, les pédagogies alternatives impliquent-elles une libéralisation et une privatisation de l'éducation ou sont-elles les laboratoires d'une transformation future de l'institution scolaire publique et les révélatrices d'une nouvelle 
façon d'enseigner?

Plusieurs axes nous sont apparus pour délimiter et analyser cette galaxie.

En premier lieu, la nécessité de définir les pédagogies alternatives dans leur variété et leur originalité mais également de les situer par rapport aux courants de l'éducation nouvelle. Leurs positionnement et leurs ambiguïtés à l'égard des courants " historiques » Freinet, Decroly, Steiner et Montessori nous semblent une clé pour mieux cerner les principes fédérateurs de cette nouvelle nébuleuse pédagogique mais aussi déceler cette « lutte de pouvoir » pour être les tenants d'une alternative pédagogique à l'enseignement dit traditionnel

En second lieu, la notion du développement personnel apparaît comme un paradigme structurant des écoles et des acteurs de ces courants. Comment percevoir la convergence des notions de bienveillance, positivité, bien-être au sein de ces pédagogies? Quelles sont les attentes d'une éducation fondée sur le développement personnel ?

Ensuite, l'essor des neurosciences dans l'éducation nous est apparu comme un axe de compréhension de l'assise "théorique» de ces pédagogies. Fort du caractère " scientiste » des neurosciences, de leurs impacts dans la société, ces écoles ne veulentelles pas "légitimer" leurs expériences éducatives par le biais d'une validation scientifique?

Bien sûr l'étude de telles formes éducatives nous impose de préciser le rôle des parents, acteurs majeurs de ces expériences.

Enfin, il convient de souligner les liens, les résistances voire les oppositions à l'institution scolaire publique. Présentes en grande partie dans l'enseignement privé, ces écoles ne participent-elles pas à une privatisation, rampante ou assumée, de l'éducation? Mais hors ou dans l'institution scolaire, l'étude des pédagogies alternatives nous incite à analyser leurs liens avec l'institution scolaire et les différentes "stratégies» d'entrisme, ou d'évitement de l'enseignement public.

Toutes ces questions, récurrentes lorsque l'on aborde une alternative pédagogique, soulignent l'importance de la question des finalités revendiquées, assumées et réelles de ces pédagogies.

\section{Des écoles différentes aux pédagogies alternatives?}

Comment est-on passé d'une terminologie d'écoles «différentes » à celle apparue au début des années 2010 de "pédagogies alternatives»? Cette évolution radicale des termes n'illustre-t-elle pas un changement de paradigme? Si les écoles "différentes » revendiquent leurs filiations à l'égard des pédagogies dites d'éducation nouvelle, qu'en est-il des pédagogies alternatives? Les pédagogies dites alternatives actuelles sont-elles les résurgences directes des pédagogies d'éducation nouvelle du début du XXe siècle?

\section{Une mutation sémantique et politique}

17 La terminologie d'écoles différentes a été développée par les travaux respectifs et communs de Marie-Anne Hugon et Marie-Laure Viaud (Viaud \& Hugon 2015 ; Viaud 2005, 2015, 2017). En 2005, en étudiant une trentaine d'établissements de l'enseignement public et privé, Marie-Laure Viaud avait donné une définition très " souple " puisqu'il s'agissait des «classes et écoles qui, à tous les niveaux scolaires, sont repérés dans le paysage éducatif 
comme alternatives, expérimentales et/ou se réclamant du courant des pédagogies nouvelles " (Viaud, 2005, p.32).

Marie-Anne Hugon s'intéresse tout particulièrement aux écoles "différentes " qui tentent d'expérimenter d'autres façons d'enseigner au sein de l'éducation nationale. Elle note le faible nombre des établissements qui se réclament de pédagogies différentes : 9 collèges et lycées et 23 écoles Freinet. Marie-Anne Hugon et Marie-Laure Viaud précisent la difficulté de repérer dans le paysage éducatif des écoles aux "pratiques différentes » mais aussi, pour ces écoles, d'avoir une lisibilité. Les établissements secondaires répertoriés (micro-lycées, pôle innovant lycéen ou collège Decroly) se retrouvent au sein de la Fespi (fédération des établissements scolaires publics innovants), association qui devait permettre cette visibilité au sein du paysage éducatif français.

Une ligne de clivage pédagogique et idéologique est perceptible, certains établissements comme le Lycée autogéré de Paris ou l'école Vitruve affichant explicitement une volonté de transformation sociale et politique et d'autres, comme les micro-lycées, étant plus une réponse institutionnelle et technique au décrochage scolaire qu'une réelle volonté critique du système éducatif auxquels ils appartiennent par ailleurs.

Ainsi la terminologie de pédagogies et écoles différentes souligne surtout la volonté d'équipes de travailler, différemment mais au sein de l'enseignement public.

Depuis 2010, le terme de pédagogies "alternatives» est utilisé surtout pour des structures, privées. L'idée d'alternative se situant par opposition à l'enseignement public. Quelle est la nature de cette opposition? Il s'agit pour ces structures de repenser le rythme de l'enfant, son statut, le rôle de la parentalité avant même la question de la scolarisation. La première complexité pour appréhender ces écoles vient de la variété des projets pédagogiques et politiques. En effet, il apparaît difficile de mettre dans le même courant de pensée les écoles religieuses intégristes, les écoles Montessori, les écoles dites démocratiques et les militants de l'instruction à domicile.

\section{Pédagogies nouvelles et " pédagogies alternatives » : même combat?}

L'idée d'alternative n'est pas nouvelle puisque les courants d'éducation nouvelle issus des pédagogues Célestin Freinet, Maria Montessori, Rudolf Steiner et Ovide Decroly sont présents en France depuis la première moitié du XXe siècle et se sont structurés en réaction à l'école traditionnelle. Les courants d'éducation nouvelle, comme les actuelles pédagogies alternatives, ne sont aucunement des courants structurés et cadrés mais bien des nébuleuses de pensées plurielles. Au début du XXe siècle, les courants d'éducation nouvelle ont revendiqué une "révolution copernicienne », une réorganisation scolaire et des méthodes d'enseignement, une révolution éducative dans et hors l'école, à travers une autre vision de l'enfant et d'autres relations pédagogiques entre adultes et enfants. Les pédagogies nouvelles nées au XXe siècle et les pédagogies alternatives du XXIe siècle apparaissent différentes dans leurs finalités politiques, leurs modalités et leurs assises scientifiques.

24 Premièrement, les pédagogies alternatives se dissocient des pédagogies nouvelles par cette volonté de ne pas se laisser «enfermer» dans une pédagogie spécifique Freinet, Decroly, Steiner et même Montessori; car cette dernière est une référence et un « esprit». Si ces écoles entendent "s'inspirer» de ces pionniers, elles développent 
d'autres concepts comme ceux du «bien être », du développement personnel et du rôle des neurosciences.

Deuxièmement, les finalités politiques semblent très éloignées. Les mouvements Freinet mais aussi dans une moindre mesure Decroly revendiquent une finalité émancipatrice et de transformation politique. Les mouvements de pédagogies alternatives se revendiquent le plus souvent "apolitiques» et soulignent avant tout le développement de la personnalité et le développement personnel. Même si les deux éléments ne sont pas nécessairement contradictoires, une césure idéologique est perceptible entre ces différents groupes. Cette dépolitisation déclarée est toutefois " politiquement située ». En effet, cette affirmation "d'apolitisme» est une façon de ne pas se rattacher idéologiquement et directement à un courant politique précis et implicitement daté. Cependant, ce refus d'être " étiquetées " n'empêche pas un discours et des pratiques qui renvoient à des univers politiques et pédagogiques bien identifiés.

Toutefois, le réseau des écoles privées hors contrat dites « indépendantes » soutenues par la fondation pour l'école dirigée par Anne Coffinier représentent un courant qui assume une logique d'opposition à l'école publique actuelle et qui prône un retour à une école « classique » au même titre que le réseau espérance-banlieue (Chambat, 2018) et les écoles de la fraternité Pie X (Damgé, 2018). De leur côté, les écoles Don Bosco et Saint-Jean Baptiste de la Salle, tout en revendiquant le caractère confessionnel de leurs pédagogies, prônent des pédagogies différentes autour d'un projet éducatif spécifique ${ }^{2}$.

èmement, leurs assises scientifiques ne sont pas les mêmes. Les courants d'éducation nouvelle sont issus des réflexions et des pratiques de la psychologie de l'enfant et de la psychologie sociale. Les pédagogies alternatives, tout en reprenant une partie du corpus psychologique légitiment leurs pratiques par les avancées des sciences cognitives et en particulier les neurosciences.

Le Printemps de l'éducation, diffuseur de la diversité des idées des pédagogies alternatives, propose un petit lexique des pédagogies "différentes", repris par les autres sites de diffusion favorables aux pédagogies alternatives. ${ }^{3}$

Ce petit lexique place dans l'ordre : la pédagogie Montessori, Steiner, Freinet, pédagogie institutionnelle, éducation intégrale et libre progrès, éducation lente, modèle scandinave, éducation démocratique, école à la maison, éducation authentique. ${ }^{4}$

Mettre sur le même plan ou dans la même nébuleuse, la pédagogie Freinet, aux fondements émancipateurs et politiques précis et structurés et l'éducation ésotérique du libre progrès de Mirra Alfassa soulève de nombreuses interrogations. Le rapport Miviludes publié en avril 2015 a d'ailleurs noté que le site du Printemps de l'éducation diffusait les idées d'un panel très hétéroclite de mouvements et acteurs éducatifs ayant peu de choses en commun (Miviludes, 2013).

31 Un autre diffuseur actif des pédagogies alternatives, le Festival de l'école de la vie est symptomatique par son action au sein des réseaux sociaux d'une volonté d'amalgamer toutes les questions d'éducation, de parentalité et de bien-être. On trouve ainsi dans le site, des propositions sur les familles parentales, la garde partagée, des questions sociétales mais aussi des éléments sur l'astrologie et toutes sortes de spiritualités ${ }^{5}$.

Un des pôles unificateurs de ces courants et diffuseurs est le mouvement Colibris, inspiré par Pierre Rahbi. Le mouvement propose sur son site toute une série d'informations sur les pédagogies alternatives, de «kit » de création d'un « lieu d'éducation alternatif » et d'ouvrages qui s'éloignent clairement de l'éducation nouvelle ${ }^{6}$. Le mouvement a insufflé 
la création d'écoles comme celles des Amandins ou Isabelle Pelloux diffuse une pédagogie fondée sur la coopération (Pelloux, 2014). Sophie Rahbi, qui se déclare formatrice Montessori est une conférencière active dans le domaine de l'éducation.

Les éditions Actes Sud sont l'un des principaux éditeurs des publications de ces expériences. On retrouve ainsi les écrits d'André Stern, militant de l'instruction à domicile ou de Ramïn Farhangi, cofondateur de l'école dynamique de Paris, mais aussi les écrits en français des penseurs des écoles « démocratiques » comme Peter Gray (Sterne, 2011 \& 2017; Farhangi, 2018; Gray, 2016). Françoise Nyssen, codirectrice des éditions Actes sud est elle-même cofondatrice de l'école à pédagogie alternative proche du courant Steiner en 2015, l'école du domaine du possible.

Les militants de pédagogies alternatives et autres «conférenciers» de cette nébuleuse rappellent, souvent le rôle des pionniers de l'éducation nouvelle pour mieux les cantonner au passé. De leur côté, les mouvements de l'éducation nouvelle comme Freinet ou Decroly ne cherchent pas à être amalgamés à des courants ésotériques ou qui proposent un contournement de l'enseignement public. La situation est plus fluctuante en ce qui concerne les courants Montessori et Steiner qui illustrent une grande porosité avec tous ceux qui prônent une autre façon de faire hors de l'enseignement public.

Ainsi, des choix idéologiques sont perceptibles derrière ces conceptions éducatives: finalités de l'éducation, conception de l'enfant, conception de l'organisation éducative, conception des relations adulte-enfant, conception des apprentissages, des programmes et des méthodes pédagogiques.

\section{Les courants historiques de l'éducation nouvelle}

\section{Des principes fédérateurs}

36 Les pédagogies alternatives du XXIe siècle, qu'elles soient hors ou dans l'école, se revendiquent implicitement ou explicitement des courants d'éducation nouvelle du début du XXe siècle (Montessori, Decroly, Freinet, Steiner). Ces expériences éducatives alternatives sont parfois des émanations directes de ces pédagogies comme c'est le cas pour les écoles Montessori ou les classes Freinet

Changer l'éducation, c'est le projet des pédagogues de l'éducation nouvelle. L'idée d'une «Éducation nouvelle » n'est pas nouvelle, même au début du 20ème siècle. Elle plonge ses racines au moins dans le 18ème siècle avec Rousseau puis Pestalozzi et elle s'épanouit à la fin du 19ème siècle, au début du 20e siècle avec Maria Montessori ou Célestin Freinet. Le projet de renouveler de fond en comble l'entreprise éducative est donc un fait historique de longue durée, caractéristique des sociétés occidentales contemporaines et de leur projet démocratique.

38 Mais quels sont schématiquement les principes de l'Éducation nouvelle?

En premier lieu, priorité est donnée à l'éducation, à rebours de l'acquisition d'une masse de connaissances. Une primauté de l'éducation sur la seule transmission des savoirs et de l'instruction mais également la définition d'une éducation qui se veut intégrale, c'est à dire pas seulement intellectuelle, mais aussi morale, physique et affective. En second lieu, l'action éducative doit prendre en compte la singularité de l'enfant en tant que tel. L'enfant n'est pas un homme en miniature, il doit pouvoir vivre sa vie d'enfant. En troisième lieu, l'éducation est centrée sur l'enfant, non sur les savoirs ou le maitre. La 
pédagogie » classique » est centrée sur la préparation de l'homme de demain, alors que l'école active considère que l'éducation est une vie avant d'être une préparation à la vie. Par l'éducation fonctionnelle centrée sur les intérêts de l'enfant, il s'agit de s'adapter aux besoins de l'enfant, et non de déterminer ce qu'il est utile de faire d'apprendre, les habitudes à faire contracter, les exercices propres à obtenir les résultats visés. L'autonomie cristallise le quatrième principe. Car l'autonomie suppose la maitrise de soi et la domination des tendances instinctives. Elle se fonde sur une base librement consentie et non pas imposée. Le cinquième principe reste que cette éducation individuelle, centrée sur le sujet se fait, doit se faire, dans un esprit communautaire. L'école nouvelle, qui promeut l'école sur mesure, la liberté des rythmes, combat l'individualisme et la docilité. Quant à l'esprit communautaire, ce n'est pas le chacun pour soi, le classement, mais la préparation à la vie sociale par la vie en commun. Enfin le dernier principe désigne un esprit, une atmosphère d'optimisme et de confiance.

L'historiographie de l'éducation nouvelle a bien montré qu'il s'agit d'une nébuleuse de courants pédagogiques qui peuvent converger sur quelques principes fédérateurs mais aussi avoir de profondes différences d'appréciation et de projets (Hameline, Helmchen, \& Oelkers, 1995 ; Peyronie, 2002 ; Avanzini, 2003 ; Ohayon, Ottavi \& Savoye, 2004). Annick Ohayon, Dominique Ottavi et Antoine Savoye dans leur ouvrage, Histoire, présence et devenir de l'Education nouvelle, soulignent le risque d'une définition réductrice et d'une éducation nouvelle unitaire :

«En fait, nous avons affaire à une nébuleuse où voisinent théories philosophiques, pratiques novatrices, idéaux politiques et où abondent les conflits. Se donner une seule définition, préalable au travail d'élucidation de la notion, relèverait d'une attitude dogmatique » (Ohayon, Ottavi, Savoye, 2004, p.26).

On perçoit néanmoins toujours des tentatives de regroupement entre ces mouvements.

Depuis 1970, l'association nationale pour le développement de l'éducation nouvelle regroupe une dizaine d'écoles privées qui se reconnaissent dans les principes de l'éducation nouvelle. Autour de l'école La Source fondée par le pédagogue Roger Cousinet (1881-1973) on retrouve l'école nouvelle d'Antony ou la Prairie de Toulouse. Les principaux objectifs de l'ANEN restent encore aujourd'hui d'accroitre les échanges entre ces écoles et promouvoir une formation pour les enseignants de ces structures.

41 Autre initiative plus récente, en octobre 2017, la biennale internationale de l'éducation nouvelle a tenté de renouer les liens existant entre les courants «historiques»de l'éducation nouvelle et les réformateurs pédagogiques de l'enseignement public :

«Différentes approches de la pédagogie existent, mais toutes ne se valent pas. Nos associations se réfèrent, au-delà de leurs différences, à un socle commun; qu'on appelle toujours l'éducation nouvelle, même si elle a une longue histoire $»^{8}$

Les mouvements organisateurs de cette biennale sont significatifs de la volonté de " réactualiser » les principes et les pratiques de l'éducation nouvelle, pratiques utilisées en partie dans leurs classes et écoles. Ces mouvements, contrairement aux autres pédagogies alternatives, revendiquent leur histoire et en font une assise de leur projet actuel :

«Il nous parait indispensable de retourner vers notre histoire, de partager ces racines communes et de mesurer ensemble l'incroyable modernité de nos projets ».

Cette biennale est à l'initiative des Céméa, du Crap-cahiers pédagogiques, de la Fespi, de l'icem. Ces structures sont de nature différente. Le GFEN, groupe français d'éducation nouvelle représente la section française créée lors de la création de la ligue internationale 
de l'éducation nouvelle qui, à partir du congrès de Calais en 1921, tente de regrouper dans des congrès successifs jusqu'à la seconde guerre mondiale, les courants se reconnaissant dans l'éducation nouvelle (Brehony, 2004).

L'ICEM, l'institut coopératif de l'école moderne, créé en 1947 représente le réseau des enseignants de la pédagogie Freinet ${ }^{9}$. Les CEMEA, centres d'entrainement aux méthodes d'éducation active, le CRAP, centre de recherche et d'action pédagogique étant également historiquement la structure de soutien aux classes nouvelles qui se développement dans les années 1950 dans l'enseignement public français, les cahiers pédagogiques étant le bulletin de liaison du CRAP. Leur objectif est bien d'actualiser les pratiques et les principes directeurs de l'éducation nouvelle :

«Dans le contexte actuel, le projet pédagogique de l'éducation nouvelle est aussi un projet politique qui trouve plus que jamais sa pertinence pour inventer des réponses adaptées aux besoins de publics les plus divers, pour donner plus de sens aux apprentissages scolaires ou informels. (...) la transformation sociale par l'éducation nouvelle reste donc un projet ambitieux, captivant, mobilisateur ! ${ }^{10}$

La difficulté de ce discours est qu'il reprend le "mythe" d'une éducation nouvelle structurée, unie dans un élan émancipateur alors que l'histoire de l'éducation nouvelle marque surtout la complexité de mouvements ou courants disparates où le projet politique n'est pas toujours explicite.

\section{Maria Montessori super star?}

La singularité de Maria Montessori (1870-1952) est d'être une figure emblématique de l'éducation nouvelle du début $d u$ XXe siècle mais aussi la référence de toutes les pédagogiques alternatives actuelles.

Fondée sur la confiance dans l'enfant, la volonté de développer chez lui une autonomie et une responsabilité, cette pédagogie connait depuis une dizaine d'années un essor important jusqu'à devenir un objet commercial de premier plan par une multitude de produits estampillés Montessori mais qui semblent éloignés des idées et propositions éducatives de Maria Montessori.

Le site Festival de l'école de la vie, grand diffuseur d'infos sur les pédagogies alternatives résume bien l'état d'esprit à l'égard de Montessori :

«Cette pédagogie qui fait ses preuves depuis une centaine d'années, et récemment mise en lumière par Céline Alvarez, se fonde sur l'éveil sensoriel de l'enfant et le développement de son esprit d'autonomie, favorisé à l'école par une atmosphère concentrée, sous les yeux d'un enseignant bienveillant. Travail individualisé, matériel autodidactique, règles de vie responsabilisantes. Une pédagogie qui respecte les rythmes naturels du développement de l'enfant, et qui le considère comme une personne digne d'intérêt et constituant l'avenir d'une société de paix » ${ }^{11}$.

49 Une tendance lourde permet au mouvement pédagogique montessorien de sortir d'une certaine marginalité pour être depuis le début du XXIe siècle au cœur de nombreuses préoccupations.

Actuellement les écoles Montessori en France sont exclusivement des écoles privées hors contrat mais un courant semble se dessiner dans l'enseignement public. Ainsi une association, «public Montessori », composée d'enseignants du public tente de développer les idées de Maria Montessori au sein de l'éducation nationale en proposant des 
formations et des ouvrages pédagogiques. ${ }^{12}$ Des formations sont proposées par les inspections locales mais aussi par certains syndicats signe tout au moins d'un intérêt. ${ }^{13}$

51 Le «cas» Céline Alvarez reflète cet intérêt grandissant pour des méthodes « alternatives ». Professeure des écoles à Gennevilliers de 2011 à 2014, Céline Alvarez a relaté son expérience pédagogique dans son ouvrage Les lois naturelles de l'enfant, bestseller qui a propulsé la jeune enseignante au rang d'icone d'une autre conception des apprentissages.

Céline Alvarez se place dans cette logique d'une pédagogie scientifique montessorienne tout en voulant «actualiser» ses idées et pratiques et ne pas seulement être une discipline de Maria Montessori (Alvarez, 2016).

Son expérience a produit un nombre important de controverses, sur la véracité de son discours, sur l'objectivité des résultats, sur l'évaluation « scientifique » de son travail, sur la nature de ses soutiens. En particulier, le soutien financier de l'institut Montaigne et moral du neuroscientifique Stanislas Dehaene aux travaux et discours de Céline Alvarez illustre une alliance de fait entre des associations qui prônent une «libéralisation » de l'éducation, l'impact des neurosciences en éducation et des expériences qui se veulent 《alternatives $»^{14}$. Mais ce qui nous intéresse ici c'est d'établir non pas l'analyse de son « expérience » mais son impact.

Son succès éditorial est indéniable, plus de 200.000 exemplaires vendus en mars 2018 , ouvrage traduit dans une dizaine de langues, un site prolifique, des conférences qui font salle pleine et des cycles de formation d'enseignants parfois soutenu par des institutions publiques comme en Belgique francophone ${ }^{15}$. De très nombreux enseignants du privé et du public ont montré un intérêt pour son discours et cette volonté de transformation pédagogique. Son site comme le nombre de ressources proposés ne peut qu'être noté dans ce travail de diffusion et d'influence grandissante.

Mais ce qui distingue fortement ces nouvelles pédagogies avec celles de l'éducation nouvelle, c'est l'importance donnée à la notion et aux pratiques de développement personnel.

\section{Le développement personnel, entre individualisme et émancipation, le Graal des pédagogies alternatives ?}

56 L'essor de la notion de développement personnel est un mouvement de fond depuis plusieurs décennies de nos sociétés occidentales. La volonté de développement personnel s'insère dans tous les domaines de la société comme un apprentissage à vivre: "les techniques de développement personnel visent à la transformation de soi : soit pour se défaire de certains aspects pathologiques (phobie, anxiété, déprime, timidité), soit pour améliorer ses performances (mieux communiquer, gérer son temps, s'affirmer" (Sciences Humaines, 2011).

57 Cette notion, devenue un domaine de réflexion à part entière, véhicule une terminologie très disparate, de la singularité, à l'épanouissement, au bien-être, à la bienveillance, l'empathie, la transformation de soi, l'émancipation ou la compétition et l'individualisme.

Michel Lacroix souligne le risque de l'image de la plante qui pousse, image "séduisante » et qui «paraît aller de soi » et pourtant dangereuse dans ce qu'elle véhicule l'idée d'une «préprogrammation » et de schéma préétabli. Pour le philosophe, ce n'est pas la notion 
de développement personnel qui est en jeu mais bien la façon dont on le conçoit. Une tension est perceptible entre un développement personnel défini comme une "prédestination» ou un développement qui s'apparente à un cheminement, de «multiplicité de styles d'existence » au nom de la liberté de la nécessaire réflexion sur une réalisation de soi émancipatrice (Lacroix, 2013).

\section{New Age et psychologie positive des références assumées?} pratiques, techniques, consultations, soutiens, coachings, etc. dans le domaine de l'éducation hors secteur public. » (Miviludes, 2015, p.80). Sont particulièrement visés par la mission les sites «pro-instruction à domicile de diverses orientations politiques, qui jouent sur les angoisses des parents pour promouvoir l'école à la maison, la création d'écoles alternatives etc.». La mission vise nominativement le site de l'écologie de l'éducation d'André Stern et du Printemps de l'éducation d'Antonella Verdiani. Ce dernier site place effectivement dans le même ordre que d'autres pédagogies, les théories éducatives de Mirra Alfassa (1878-1973) de l'éducation intégrale et libre progrès à Auroville dont la présidente du Printemps de l'éducation est une de spécialistes reconnues (Verdiani, 2008).

62 Mais une des racines assumées des pédagogies alternatives est dans la psychologie positive. Une psychologie qui s'intéresse au bien-être, à la réalisation de soi et qui en empruntant des méthodologies à la psychologie et aux neurosciences connait un succès grandissant. Les courants pédagogiques qui entendent développer dans les apprentissages la notion de développement personnel se réfèrent à la psychologie «humaniste» de Carl Rogers (1902-1982). Les écoles démocratiques rappellent ses apports en psychologie pour "légitimer» leurs expériences pédagogiques actuelles ${ }^{16}$. Mais c'est la psychologie positive initiée par Martin Seligman en 1998 qui semble être la référence fédératrice des pédagogies alternatives avec la prise en compte des compétences relationnelles, de l'optimisme, de la confiance et de l'estime de soi dans les apprentissages. (Seligman, 1998).

63 A partir de cette notion de psychologie positive de nombreuses associations comme Positran d'Ilona Bodiwell ou Scholavie de Laure Reynaud ont fait le choix par des formations, des conférences tout un dispositif éditorial d'axer leurs travaux sur l'éducation en gravitant dans la galaxie des pédagogies alternatives. 


\section{Bienveillance et positivité : une lutte contre la « pédagogie noire » ?}

64 La bienveillance, notion entrée récemment dans le vocabulaire de l'éducation (Brugère, 2011; Ginchard \& Petit, 2011; Molinier, 2009 ; Noddings, 2012 ; Tronto, 2009; Usclat, Hétier \& Monjo, 2016) est un autre principe fédérateur des pédagogies alternatives. Depuis la loi de refondation de l'école de la République du 13 juillet 2013, les textes officiels du ministère de l'éducation nationale soulignent que « les conditions d'un climat scolaire serein doivent être instaurées dans les écoles et les établissements scolaires pour favoriser les apprentissages, le bien-être et l'épanouissement des élèves et de bonnes conditions de travail pour tous $»^{17}$. Notion utilisée au Canada et aux Etats-Unis associée au concept de care, de bien-être, d'estime de soi, le terme de bienveillance se retrouve avec le bien-être comme des leviers au service des élèves pour une "école bienveillante face aux situations de mal-être des élèves ». (Usclat, Hétier \& Monjo, 2016).

Du point de vue scientifique, plusieurs études commencent à délimiter les contours d'un terme encore fluctuant et flou (Usclat, Hétier \& Monjo, 2016).

Si l'institution scolaire publique entend développer une "école bienveillante", les pédagogies alternatives de l'éducation nouvelle ou plus récente en font un pivot de leur raison d'être.

Fabienne Serina-Karsky a bien montré l'historicité de la notion de bien-être. Les pratiques éducatives qui favorisent le bien-être de l'enfant sont une des contributions majeures des pédagogies d'éducation nouvelle à ce qu'elle nomme un nouveau paradigme éducatif (Serina-Karsky, 2013).

Les militants des pédagogies alternatives actuelles se réfèrent tout d'abord à la psychanalyste Alice Miller (1923-2010) qui analysa les maltraitances faites aux enfants et leurs conséquences. Dans son ouvrage, C'est pour ton bien elle développe la notion de lutte contre la « pédagogie noire » (Miller, 1985) en reprenant l'argumentation de Katharina Rutschky (1941-2010). (Rutschky, 1977).

La pédagogie noire est une forme d'éducation répressive visant à soumettre les enfants par la violence et le harcèlement physique et moral. Il ne s'agit plus seulement d'un développement dans la bienveillance qui est proposé mais bien la fin d'une domination adulte. Car cette pédagogie noire est analysée comme un moyen de maintenir la hiérarchie arbitraire entre adulte et enfant, de maintenir les parents consciemment ou non dans des conflits intérieurs. La déification des adultes et la négation des sentiments et des émotions empêchent l'enfant d'être autonome et responsable.

70 La lutte contre ses rapports de pouvoir doit donc laisser place au respect, la bienveillance et l'amour, l'empathie. Des notions que l'on retrouve dans l'enseignement public. Une des auteurs phare des pédagogies alternatives dans la lignée de Miller est actuellement Catherine Gueguen. La psychologue, contrairement à Miller qui rend rédhibitoire la pédagogie noire, met en avant la faculté de résilience. Dans son ouvrage Pour une enfance heureuse, Catherine Gueguen souligne bien les mécanismes destructeurs développés par Miller mais se refuse à tout déterminisme. (Gueguen, 2014, 2015a \& 2015b). Catherine Gueguen se place résolument dans une posture croisée de cette question de résilience à l'école en mettant en avant le rôle des neurosciences "affectives et sociales" pour changer l'éducation et développant les thématiques de la psychologie positive Intervenante au séminaire national des inspecteurs de l'éducation nationale chargés de 
mission pour l'école maternelle, Catherine Gueguen mêle les recherches en neurosciences et l'analyse des applications sur le développement émotionnel et affectif de l'enfant. Ses conférences se trouvent diffusées au sein même des établissements publics, marquant ainsi très nettement un appui de l'institution scolaire à l'étude et la connaissance de ces notions. ${ }^{18}$

Très présente aussi dans les sites des pédagogies alternatives, Isabelle Filliozat se veut dans la lignée de Miller et Gueguen. Elle multiplie les ouvrages, vidéos, conférences jusqu'à proposer tout un dispositif d'ateliers qui lient psychologie positive, conseils aux parents; le tout, comme il se doit, "validé par les neurosciences ", sans que toutefois, les études précises soient référencées ${ }^{19}$.

L'attrait pour les questions de développement personnel permet aussi à toute une série de « coach» d'entrer dans le domaine de l'éducation. Le chef de file est sans conteste Ken Robinson mais par exemple nous pouvons au sein du Festival de l'école de la vie retrouver toute une pléthore de coach du développement personnel qui ne possèdent que des liens forts lointains avec l'éducation et l'apprentissage, qu'il s'agisse de David Laroche ou Jonathan Lehmann.

\section{L'impact des neurosciences}

73 Le rôle des neurosciences en éducation apparait comme un élément fédérateur de toutes les pédagogies alternatives récentes. L'essor des neurosciences dans notre société est un sujet qui questionne par l'importance qu'on prit les neurosciences dans toutes les activités humaines; mais c'est plus prosaïquement pour les pédagogies alternatives un outil de légitimation de leurs pratiques. En effet, la connaissance accrue du cerveau semble valider une partie importante de ce que nous pourrions appeler les intuitions des pédagogues d'éducation nouvelle. Néanmoins, la question n'est pas de s'opposer ou d'être fasciné par les neurosciences mais d'établir leurs liens avec l'éducation. Les pédagogies alternatives par l'importance qu'en donne une partie de militants à la validation par les neurosciences comme du rôle accru des neuroscientifiques en éducation fait de l'impact des neurosciences sur une question éducative et politique.

\section{Une légitimation scientifique par les neurosciences?}

Si les neurosciences peuvent se définir comme l'étude scientifique du système nerveux et du fonctionnement du cerveau, la neuroéducation est l'étude des mécanismes cérébraux appliqués aux problèmes de l'éducation et du comportement de l'enfant. Issue des neurosciences et du «tournant neuro » des années 1980-1990, la neuroéducation ne se dessine comme une nouvelle discipline qu'à partir de 2007 (Chamak \& Moutaud, 2014; Chamak, 2017; OCDE, 2007). Champ d'application des neurosciences en éducation, la neuroéducation est devenue le nouveau graal d'une pédagogie scientifique et innovante et une référence pour les pédagogies alternatives.

Céline Alvarez précise "il faut relire Montessori à la lumière des nouvelles découvertes en neurosciences pour réformer l'école, dont le système actuel est une aberration pour le fonctionnement de l'enfant, dans son ensemble ». (Alvarez, 2016)

76 Isabelle Filliozat dans la même logique souligne : 
"Comprendre ce qui se passe dans leur cerveau pour mieux communiquer et accomplir le rêve de tout parent: les aider à construire les fondations de leur sécurité intérieure, les accompagner dans l'intégration de leur confiance en leur personne propre comme en leurs compétences, pour qu'ils deviennent des adultes autonomes, intelligents, responsables et empathiques. $»^{20}$

L'apport des neurosciences est même défini par le site du Festival de l'école de la vie comme une mesure de santé publique :

«En plus de favoriser leurs apprentissages, faire preuve de bienveillance dans l'éducation de nos enfants est également bon pour leur santé ! En effet, les récentes découvertes en neurosciences ont démontré que l'éducation positive jouait un rôle important dans le développement d'une partie essentielle du cerveau, et qu'elle aurait aussi un effet considérable sur la mémoire $»^{21}$.

De leur côté certains neuroscientifiques comme Olivier Houdé ou Stanislas Dehaene marquent leur intérêt et leur soutien à certaines expériences de pédagogies alternatives, comme celle de Céline Alvarez, semblant donner l'impression d'une «alliance de fait » entre neurosciences et pédagogies alternatives.

Le site du ministère de l'éducation nationale donne lui-même une définition de la neuroéducation :

«discipline scientifique émergente, à la croisée des neurosciences, de la psychologie et de l'éducation, la neuroéducation se donne pour objectif de rendre compte aux éducateurs, enseignants et professeurs, des meilleures stratégies et méthodes d'enseignement, à partir des découvertes scientifiques sur la mémoire, le langage et l'apprentissage ${ }^{22}{ }^{22}$

80 Cette définition reste contestée, non pas dans l'idée même de neuroéducation, mais surtout de lien direct entre recherches en neurosciences et modification des méthodes d'enseignement et stratégies d'apprentissages.

\section{Entre fascination et répulsion}

81 La neuroéducation entend améliorer les méthodes d'enseignement en s'appuyant sur les mécanismes cérébraux d'apprentissage décrits par les neurosciences et la psychologie cognitive. Les controverses pédagogiques, conceptuelles et politiques sont parties prenantes de l'élaboration des théories du développement. (Friedrich, Hofstetter \& Schneuwly 2013)

Mais les controverses actuelles sur l'omniprésence des neurosciences et d'une démarche exclusivement scientifique de l'éducation sont l'objet d'écrits virulents qui se multiplient au moment où semble se dessiner une conjonction particulièrement favorable pour les neurosciences, avec un soutien explicite du pouvoir institutionnel et politique à tous les niveaux, mais aussi à un attrait grandissant de l'ensemble de la population pour les applications possibles de la connaissance du cerveau. La présence de Stanislas Dehaene, titulaire de la chaire de psychologie cognitive au collège de France, fervent défenseur de cette neuroéducation et président du conseil scientifique de l'éducation nationale est un symbole fort de l'influence des neurosciences au sein de l'institution scolaire.

Franck Ramus, dans son article "les neurosciences, un épouvantail bien commode», insiste sur le fait que les neurosciences suscitent à la fois fascination et répulsion. (Ramus, 2014).

84 Cette fascination est liée à la connaissance du cerveau, monde qui apparait comme une nouvelle galaxie ainsi qu'à l'ouverture vers des applications sociales et des perspectives 
vers la neuropsychologie, qui par l'étude des émotions, illustre un champ nouveau de développement de la psychologie. particulièrement notable dans l'ensemble de pédagogies d'éducation nouvelle et les pédagogies alternatives. Une des raisons est la volonté de penser l'enfant dans sa globalité, de rendre cohérent l'éducation familiale et scolaire. Mais au-delà de cette logique, les pédagogies alternatives ne sont-elles pas la revanche de parents sur l'école traditionnelle?

\section{Une pente familialiste?}



plupart des homeschoolers soulignent l'importance des apprentissages informels. Daniel Shugurensky (2017) analyse les défis conceptuels, méthodologiques de ces apprentissages. Ces défis lui permettent de préciser que les apprentissages informels, vont bien au-delà des savoirs et des compétences scolaires et qu'ils ont été sous-estimés par rapport aux apprentissages dits formels qui au contraire sont les seuls pris en compte par le système scolaire classique :

"Les apprentissages informels ont toujours existé chez l'être humain mais, à la manière

d'un iceberg, la majeure partie est immergée et invisible » (Shugurensky, 2017). 
Farhangi de l'école dynamique de Paris, l'objectif est de lutter contre « une domination adulte » au profit d'un essor de la liberté de l'enfant qui lui permettra de s'épanouir.

\section{Refus d'école ou refus de l'école traditionnelle ? l'exemple de l'éducation à domicile}

102 Le homeschooling est bien sur un exemple radical d'alternative pédagogique à l'école, mais l'instruction à domicile en France questionne à plus d'un titre la notion de refus d'école. Depuis la loi du 28 mars 1882 sur l'obligation de l'instruction en France, la possibilité a été laissée aux familles d'instruire à domicile. Le nombre de familles qui refuse le paradigme scolaire s'accroit mais reste, avec quelques milliers d'enfants, extrêmement marginal. Ce qui nous intéresse ici c'est la définition du homeshooling et son influence au sein des pédagogies alternatives.

L'un des premiers paradoxes concernant ce phénomène est le décalage entre le nombre réduit d'enfants non-scolarisés et l'impact médiatique. Ainsi, à travers l'instruction à domicile n'y a-t-il pas une interrogation plus vaste sur l'évolution de la société dans sa globalité à l'égard de l'école?

Ensuite, peut-on parler d'une forme éducative alternative, comme semblent le présenter les associations et militants de l'instruction à domicile?

Les chiffres marquent un phénomène grandissant mais très marginal puisqu'en 2015 on comptait 24.851 enfants non scolarisés. (MEN 2016 \& Miviludes 2015, p.55). Ce nombre regroupant à la fois les scolarisés au CNED (15.051 en 2015), les scolarisés dans des établissements à distance (2.400 en 2015) et l'instruction en famille (7.400 en 2015).

En 2007-2008, les enfants non-scolarisés représentent 0,16\% des enfants soumis à l'obligation scolaire. Ils sont $0,30 \%{ }^{23}$ en 2014-2015.

L'analyse des contrôles et inspections comme des associations «non-sco » permettent une typologie non pas cloisonnée mais très fluctuante (Quatrevaux, 2011).

La méfiance de l'institution scolaire à l'égard de l'instruction à domicile amène un renforcement des dispositions de contrôle depuis avril 2018. (JO, 2018) Si l'objectif de ce projet de loi est bien de répondre à l'essor d'établissements hors contrats, les militants de l'instruction à domicile apparaissent comme des victimes collatérales des débats et de l'amalgame dans les textes ministériels entre écoles privées hors contrat et instruction à domicile.

La vigilance de l'Etat souligne l'inquiétude face à une évolution des familles qui font le choix de l'instruction à domicile dans leur refus du paradigme scolaire. Le terme d'instruction à domicile, sous-tend un repli sur la cellule familiale et une emprise des parents sur leurs enfants. C'est un enjeu de taille pour l'Etat garant de l'intérêt de l'enfant mais aussi pour les familles afin de faire comprendre qu'ils ne souhaitent pas un « repli sur soi » en créant des espaces de socialisation pour leurs enfants.

110 Il n'en reste pas moins que les débats récurrents sur le renforcement des contrôles des écoles hors contrat et instruction à domicile cristallisent l'opposition entre l'institution scolaire publique et les tenants de la non-scolarisation.

111 Le site "Printemps de l'éducation ", très actif, au sein de l'enseignement hors contrat mais aussi instruction à domicile (Miviludes, 2015), synthétise bien les critiques vis à vis de l'école en tant que telle mais aussi de l'école publique en particulier : 
"Très répandu aux Etats-Unis, le courant de l'école à la maison suscite aussi beaucoup d'intérêt en France. Ce phénomène est grandissant, notamment à cause de l'incapacité de l'école publique à se concentrer sur les besoins et les rythmes des enfants. En France, cela est possible car l'école n'est pas obligatoire, c'est l'instruction qui l'est. Le code de l'éducation prévoit cette liberté, à condition de faire une déclaration annuelle à l'inspection académique qui prévoit des contrôles pédagogiques. Pour les parents qui l'ont adoptée, il s'agit d'une part de dire non à un système qui ne convient pas, et d'autre part de préserver ce lien privilégié avec leur enfant en se chargeant eux-mêmes de leur instruction. Ils s'assurent ainsi qu'il s'épanouira à travers un cadre bienveillant et une communication non-violente tout en respectant son rythme d'apprentissage. $»^{24}$

tre une alternative semble être le souhait des militants d'instruction à domicile. Dépassant le seul refus de l'école, ils entendent être les tenants de nouvelles pratiques éducatives. (Jacquemin \& Schlemmer, 2011). Nous pouvons ici esquisser trois vecteurs de ce combat: la force de diffusion des associations "non-sco", l'objectif d'apparaitre comme les dépositaires de la « liberté pédagogique » et enfin d'être le fer de lance d'une nouvelle façon de concevoir les apprentissages.

Le début des recherches scientifiques en France sur la question permet aux tenants de l'instruction à domicile d'être « un objet d'étude » (Terrillon, 2002 ; Walter, 2005 ; Plavis, 2013 ; Delpech, 2016) mais c'est surtout la force de communication des associations et des lobbys pro-instruction à domicile qui illustre la présence des «non-sco » dans l'espace médiatique.

114 La multitude des associations, dont la représentativité est complexe à établir sont présentes dès la fin des années 1980 avec l'association Les enfants d'abord puis LAIA (Libres d'Apprendre et d'Instruire Autrement) on assiste à un nombre accru d'associations depuis 2011: «Choisir d'Instruire Son Enfant», «Parents Instructeurs de France », l'Union Nationale pour l'Instruction et l'Épanouissement (UNIE), Collectif l'école à la maison ${ }^{25}$, Collect'IEF.

115 Toutes ces associations ont le même objectif : rassembler des familles non-scolarisantes en les aidant à échanger pour pratiquer l'instruction parentale, permettre des échanges et « défendre le droit au libre choix du mode d'instruction ».

116 A côté de ces associations et en lien direct avec elles, des maisons d'édition comme les éditions instant présent sont des soutiens actifs, en précisant avoir «à cœur de promouvoir le parentage proximal, respectueux des besoins des enfants et des parents ", éditant ou rééditant les ouvrages phares de l'instruction à domicile. ${ }^{26}$ Enfin, des sites comme Printemps de l'éducation ${ }^{27}$ ou le Festival de l'école de la vie ${ }^{28}$ diffusent abondamment sur les réseaux sociaux les idées des familles «non-sco ».

117 Ensuite, tous ces groupes entendent être les tenants de la « liberté pédagogique » et sont parties prenantes de combats contre le renforcement des contrôles à leur égard depuis ces dernières années. La création des associations ces dernières années sont souvent le fruit des combats menés contre ces renforcements administratifs de l'institution scolaire. Mais peut-on parler dans ce refus d'école d'un contournement de l'école publique ou s'agit-il d'une volonté de créer une autre façon de concevoir l'éducation? 


\section{Une offensive contre l'école publique?}

118 du marché éducatif et d'une privatisation de l'éducation, il convient de noter que cette logique n'est pas structurelle. Existe-t-il une tension entre un enseignement public, commun définissant l'éducation au sein de l'école comme un bien commun, un droit fondamental pour tous et des écoles alternatives, « cheval de Troie » de la privatisation de l'éducation et du repli sur soi et les siens. Un tel manichéisme, perceptible dans les débats et discours médiatiques, est un obstacle à la compréhension des pédagogies alternatives. Il est complexe pour les militants de l'éducation nouvelle, courant « historique » de dénier aux "nouvelles" pédagogies alternatives une réelle volonté de transformer le système éducatif. Mais il est tout aussi complexe aux partisans des pédagogies alternatives de ne pas voir, ou assumer ce contournement de l'enseignement public au mépris de certaines de leurs valeurs notamment le refus de la ségrégation. L'estompage des frontières entre le privé et le public, parfois assumé par certains acteurs influents de l'éducation nationale est une réalité. Les interférences de plus en plus fortes amènent à ce qu'Yves Dutercq (2011) nomme non seulement la privatisation de l'éducation mais la 
privatisation en éducation avec l'entrée dans le système éducatif de modes de fonctionnement, de gestion, de langage, d'administration empruntés au secteur privé.

Les termes de réalisation de soi, de développement personnel, d'autonomie, de contrats d'objectifs en font partie. La notion d'autonomie est bien devenue un terme générique que chacun revendique mais qui ne sera que le résultat du rapport de force entre ceux qui $\mathrm{y}$ voient une simple notion de développement personnel et de réalisation de soi et ceux qui en perçoivent aussi l'interaction fondamentale et nous pourrions presque dire organique avec la responsabilité. Il s'agit bien d'une question ancienne mais toujours d'actualité.

Le dilemme des réformateurs de l'intérieur est qu'ils peuvent apparaitre comme ceux qui ont échoué à transformer le système et sont devenus des « conservateurs » du système en restant à l'intérieur. Le choix du ministère de propulser la pédagogie Montessori comme un nouveau graal, de faire des neurosciences l'alpha et l'Omega de toutes les réformes, rend intenable la position de ceux qui veulent réformer de l'intérieur sur les bases de l'éducation nouvelle. En cela les pédagogies alternatives deviennent des leviers pour la privatisation et pour le dénigrement de l'enseignement public.

\section{Créer sa propre école}

Une des illustrations de cette "privatisation » est l'idée même de créer sa propre école. C'est une des voies proposées par certains mouvements. Le site des colibris fournit ainsi une série d'étapes, reprise par de nombreux autres $\operatorname{sites}^{29}$ pour programmer la création d'un « lieu d'éducation alternatif ».

Le site rappelle qu'une école alternative se veut "différente" dans sa façon d'appréhender l'éducation, le rôle de l'enfant dans les apprentissages et le choix de sa pédagogie. Malgré leurs différences et spécificités, ce qui doit les unir est le but commun : "le bonheur et l'épanouissement des enfants " ${ }^{30}$. Un site dédié ${ }^{31}$ propose ainsi les démarches afin de déterminer le projet d'école et les choix pédagogiques, la nécessité d'établir une étude de marché, de préparer un «business plan» (nombre de classes, restauration, équipe pédagogique, transport, locaux, matériel etc.), de former un comité de soutien pour solliciter des contributions, de choisir la forme juridique adéquate, choisir les critères de recrutement du personnel éducatif, avant de faire la déclaration d'ouverture de l'école en tant que tel.

Dans la même logique, le mouvement des écoles démocratiques se montre très actif dans les créations, nous ne dirions pas d'écoles en tant que telles mais d'entités éducatives. Ramin Farhangi, fondateur de l'école dynamique de Paris, actif conférencier, propose un kit pour créer son école démocratique ${ }^{32}$. Ces "micro écoles» de quelques enfants semblent être une passerelle, une forme éducative pour les familles non scolarisées qui souhaitent des activités « collectives ».

\section{Conclusion}

La cartographie des pédagogies alternatives met en avant les relations et ramifications de cette galaxie. Trois archipels se détachent de notre analyse.

131 Le premier archipel est celui des courants historiques de l'éducation nouvelle. Cet archipel se structure par des pédagogies identifiables (Decroly, Montessori, Steiner ou 
Freinet). Forts des expériences pédagogiques d'écoles depuis le début du XXe siècle, ces courants sont bien présents aujourd'hui dans le paysage éducatif tout en étant marginaux numériquement. Néanmoins, si les valeurs de l'éducation nouvelle fédèrent ces mouvements, de nombreuses différences existent. Il ne s'agit aucunement d'un «front » commun. Les idéaux de mixité sociale et de transformation de l'éducation restent des points d'ancrage forts dans la mouvance Freinet et Decroly alors que les courants Steiner et Montessori mobilisent avant tout le développement de la personnalité. Ces deux derniers courants sont donc en phase avec les nouvelles écoles alternatives du début du XXIe siècle

Le second archipel, idéologiquement opposé aux idées de l'éducation nouvelle, propose un projet pédagogique centré sur la "tradition", sur la transmission des savoirs avant toute socialisation. Cet ensemble est principalement catholique traditionnaliste. Ces écoles, tout en souhaitant apparaitre comme alternatives, ne possèdent aucun lien avec les réseaux d'éducation nouvelle et restent idéologiquement en opposition avec la plupart des écoles alternatives prônant une transformation sociétale. Néanmoins, cette mouvance perçoit dans certaines écoles alternatives, une convergence dans ce contournement de l'enseignement public et dans cette libéralisation de l'éducation.

133 L'archipel central qui personnifie ce que l'on nomme aujourd'hui les pédagogies alternatives est une nébuleuse regroupant sous cette terminologie tout un ensemble d'expériences pédagogiques, d'associations et d'acteurs qui utilisent le triptyque des familles, des neurosciences et du développement personnel. Son essor reste quantitativement négligeable mais médiatiquement et politiquement très offensif. La volonté de transformation de l'enseignement "classique» et une redéfinition de l'éducation semble répondre à une aspiration grandissante de certaines franges de la population française.

134 L'un des aspects nouveau est cette captation de l'idée d'innovation par des écoles ou expériences éducatives qui ne s'attachent pas à une pédagogie spécifique ni à une forme éducative particulière. Le rôle de familles devient primordial. Paradoxalement, on assiste dans ces courants alternatifs à une sorte de tiraillement entre un repli individualiste perceptible et une volonté d'agir pour transformer le système en place.

135 Historiquement, les pédagogies d'éducation nouvelle, avec des pédagogies identifiables, structurées étaient les pédagogies alternatives. On assiste à une substitution vers des expériences qui peuvent plus ou moins se référer à des pédagogues d'éducation nouvelle. Néanmoins, le refus de se structurer par une pédagogie spécifique semble être aussi le corolaire d'un refus de pédagogues le plus idéologiquement marqués et en particulier Freinet. Ces alternatives éducatives semblent à la recherche de nouvelles figures. Ainsi, si Montessori et Steiner sont des figures revendiquées, apparaissent de nouveaux noms, comme Daniel et Hanna Greenberg pour les écoles démocratiques par exemple.

La question des finalités de ces pédagogies apparait au cœur de la possible évolution de cette galaxie. En effet, le système éducatif public français assume encore un idéal de transmission d'une culture commun, d'un "creuset» commun». Dans les faits, la situation est plus complexe et les critiques sont nombreuses, de tous bords, sur la réalité de cette finalité.

137 Actuellement, les pédagogies alternatives, telles que nous les avons définies, mettent à mal cette finalité. Elles semblent même dans une autre approche sociétale, celle de l'accomplissement de la personnalité avant toute chose. Le défi majeur pour l'institution 
scolaire, comme pour les pédagogies alternatives, n'est-il pas de faire cohabiter un idéal d'épanouissement de la personnalité, à partir des besoins et des intérêts des enfants, sans pour autant oublier l'idéal de mixité sociale, de bien commun et d'émancipation collective?

\section{BIBLIOGRAPHIE}

Avanzini, G. (2003). Scientificité, axiologie et argumentation chez les théoriciens de l'Éducation Nouvelle. Revue française de pédagogie. p.53-59.

Bonnardel, Y. (2015). La Domination adulte. L'oppression des mineurs. Paris : Myriadis.

Brehony Kevin, J. (2004). A new education for a new era: the contribution of the conferences of the NewEducation Fellowship to the disciplinary field of education 1921-1938. Paedagogica Historica. 40. 733-755.

Brugère, F. (2011). L'éthique du care. Paris : Presses Universitaires de France.

Cavallera, H. (2006). Rudolf Steiner tra antroposofia e educazione. Lecce : Edizioni Pensa, Multimedia.

Chamak, B. \& Moutaud, B. (2014). Neurosciences et société : enjeux des savoirs et pratiques sur le cerveau. Paris : A. Colin

Chamak, B. (2017). Neuromarketing : une fusion suspecte. Dans S. Lemerle \& C. Reynaud-Parigot, La biologisation du social : discours et pratiques. Nanterre : PUN.

Chambat $\mathrm{G}$ (2018). Espérance à tout prix. Cahiers pédagogiques. n547. Octobre.

Champion F. (1995). La Nébuleuse New Age. Études, 14.

Damgé Mathilde (2018). Hors contrat : comment comprendre le succès des écoles alternatives ? En ligne : Le Monde 31 aout. https://www.lemonde.fr/les-decodeurs/article/2018/08/31/hors-contratcomment-comprendre-le-succes-des-ecoles-alternatives_5348308_4355770.html

Delpech. M. (2016). Comprendre un choix éducatif méconnu : l'unschooling. Mémoire de 3eme année de Licence de sciences de l'éducation. Université de Bordeaux.

Duterq Y. (2011). Où va l'éducation entre public et privé ? Bruxelles : De Boeck.

Farhangi, R. (2018). Pourquoi j'ai créé une école où les enfants font ce qu'ils veulent. Arles : Actes Sud. Ferreux, M-J. (2001). Le New Age, ritualités et mythologies contemporaines. Paris : l'Harmattan.

Friedrich, J., Hofstetter, R. \& Schneuwly, B. (2013). Une science du développement humain est-elle possible? Controverses du début du XXe siècle. Rennes : PUR.

Gilligan, C. (2008). Une voix différente. Pour une éthique du care (A. Kwiatek, trad.). Paris :

Flammarion.

Ginchard, P. \& Petit, J.-F. (Dir.), (2011). Une société de soin, Santé, travail, philosophie, politique. Ivry sur Seine : Éditions de l'Atelier

Gray, P. (2016). Libre pour apprendre. Arles : Actes Sud. 
Gueguen, C. (2015a). Heureux d'apprendre à l'école. Comment les neurosciences peuvent changer l'éducation. Paris : Les arènes \& Robert Laffont.

Gueguen, C. (2015b). Vivre heureux avec son enfant. Un nouveau regard sur l'éducation au quotidien grâce aux neurosciences affectives. Paris : R. Laffont.

Gueguen, C. (2014). Pour une enfance heureuse : repenser l'éducation à la lumière des dernières découvertes sur le cerveau. Paris : R. Laffont.

Hameline, D., Helmchen, J. \& Oelkers, J (Dir.). (1995). L'Education nouvelle et les enjeux de son histoire. Actes du colloque international des archives IJJR (Genève 1992). Berne : Peter Lang.

Jacquemin, M. \& Schlemmer, B. (2011). Introduction : Les enfants hors l'école et le paradigme scolaire. Cahiers de la recherche sur l'éducation et les savoirs, 10. 7-28.

JO. LOI n 2018-266 du 13 avril 2018 visant à simplifier et mieux encadrer le régime d'ouverture et de contrôle des établissements privés hors contrat. En ligne : https://www.legifrance.gouv.fr/eli/ loi/2018/4/13/MENX1805338L/jo/texte

Lacroix, M. (1996). L'Idéologie du New Age : un exposé pour comprendre, un essai pour réfléchir. Paris : Flammarion.

Lacroix, M. (1995). La Spiritualité totalitaire. Le New Age et les sectes. Paris : Plon.

Lacroix, M. (2013). Philosophie de la réalisation personnelle. Paris : R. Laffont.

Lise, I. (2017). Faire l'école à la maison: Connaître le cadre légal, les outils disponibles et l'organisation à mettre en place. Paris : Editions Instant présent.

MEN. Projet de circulaire du MEN, 9 juin 2016, http://cache.media.education.gouv.fr/file/06__juin/43/7/DP-Garantir-le-droit-a-l-education-pour-tous-les-enfants-dans-le-respect-desvaleurs-de-la-Republique-et-de-la-liberte-de-l-enseignement_591437.pdf

Miller, A. (1985). C'est pour ton bien. Paris : Aubier.

Miviludes, Mission interministérielle de vigilance et de lutte contre les dérives sectaires, rapport 2013-2014 au premier ministre, 29 avril 2015.

Molinier, P., Laugier, S. \& Paperman, P. (2009). Qu'est-ce que le care ? : Souci des autres, sensibilité, responsabilité. Paris : Petite Bibliothèque Payot.

Noddings, N. (2012). “The caring relation in teaching”. Oxford Review of Éducation, XXXVIII-6, Dec.2012, 771-781.

Nozarian, B (21017). Apprendre sans aller à l'école : choisir le Homeschooling. Paris : Nathan

OCDE (2007). Rapport. Comprendre le cerveau : naissance d'une science de l'apprentissage. En ligne : https://www.oecd.org/fr/sites/

learninginthe21stcenturyresearchinnovationandpolicyapprendreauxxiesieclerechercheinnovationetpolitiques/40583325.pdf

Ohayon, A., Ottavi, D., Savoye, A. (dir.). (2004). L'Education nouvelle, histoire, présence et devenir. Bruxelles : Peter Lang.

Pelloux I. (2014). L'école du colibri : la pédagogie de la coopération. Arles : Actes Sud.

Peyronie, P. (ed.).(2002). 1922-2002, pour l'ère nouvelle et l'Education nouvelle 80 ans après. Les sciences de l'éducation pour l'ère nouvelle, 4, vol 35.

Plavis, M. (2013). Education et Ecologie. Mémoire M1 Philosophie. Université de Paris-Nanterre.

Plavis, M. (2017). Apprendre par soi-même, avec les autres, dans le monde, L'expérience du unschooling. Paris : Myriais. 
Plenel E.(1985). La République inachevée. L'État et l'École en France. Paris : Payot.

Prost, A. (1968). L'enseignement en France (1800-1967). Paris : Armand Colin.

Quatrevaux, A. (2011). Le système scolaire face à l'instruction dans la famille. Cahiers de la recherche sur l'éducation et les savoir, 10. 29-43.

Ramus, F. (2014). Les neurosciences, un épouvantail bien commode. Cités.4. 60, p. 53-70.

Rizvi, F. (2016). Privatisation de l'éducation : tendance et perspectives. Réflexions thématiques, recherche et prospective en Education, Unesco, 18, octobre.

Rutschky, K. (1977) Schwarze Pädagogik. Quellen zur Naturgeschichte der bürgerlichen Erziehung. Frankfurt am Main : Erstausgabe.

Schugurensky, D. (2007). Vingt mille lieues sous les mers : les quatre défis de l'apprentissage informel. Revue française de pédagogie, 160. 13-27.

Sciences humaines, Apprendre à vivre. Des philosophies antiques au développement personnel, Les Grands dossiers des Sciences Humaines, n² 23, juin-juillet-août 2011, p. 76.

Seligman, M. (1998). Building human strength : psychology's forgotten mission. APA Monitor, volume $29, \mathrm{n}^{\circ} 1$, janvier.

Serina-Karsky, F. (2013). Pratiques éducatives et bien-être de l'enfant à l'école : la contribution de l'Éducation nouvelle (1910-2010) : pour un nouveau paradigme éducatif. Thèse de doctorat en Sciences de l'éducation. Université de Paris 8.

Stern A. (2011). Et je ne suis jamais allé à l'école. Arles : Actes Sud.

Stern A. (2017). Jouer. Arles : Actes Sud.

Terrillon. N. (2002). L'instruction dans la famille comme alternative à l'école. Mémoire de DHEPS. Université de Lyon 2.

Topalov Ch. (1999). Les 'réformateurs' et leurs réseaux. In Ch. Topalov (dir.), Laboratoires du nouveau siècle. La nébuleuse réformatrice et ses réseaux en France, 1880-1914. Paris : EHESS, p. 13.

Tronto, J. (2009). Un Monde vulnérable. Pour une politique du care. Paris : Éditions La Découverte

Usclat, P., Hétier, R., \& Monjo, R. (2016). Le care en éducation : quelle(s) reconfiguration(s) ?

Éducation \& socialisation, $\mathrm{N}^{\circ}$ 40, En ligne : https://edso.revues.org/1482

Verdiani, A. (2008). L'éducation à la joie : un exemple d'éducation intégrale dans les écoles d'Auroville (Inde). Thèse de doctorat en sciences de l'éducation. Université de Paris 8.

Verdiani, A. (2012). Ces écoles qui rendent les enfants heureux. Arles : Actes Sud.

Verdiani, A. (2017). Renouer avec la Joie de l'enfance. Paris : Eyrolles.

Viaud, M-L. (2017). Montessori, Freinet, Steiner... Une école différente pour mon enfant ? Paris : Nathan.

Viaud, M-L. (2005). Des collèges et lycées différents. Paris : PUF.

Viaud, M-L. \& Hugon, M-A.(dir.) (2015). Les établissements scolaires « différents » et la recherche en éducation. Problèmes méthodologiques et épistémologiques. Arras : Artois Presses université

Viaud, M-L. (2015). Les innovateurs silencieux. Histoire des pratiques d'enseignement à l'université des années 1950 à 2010. Grenoble : Presses Universitaires de Grenoble

Walter, E. (2005). Qui a eu cette idée folle un jour d'inventer l'école ?, l'instruction en famille en France. Master 1 Sciences de l'éducation. Université de Lille 3. 


\section{NOTES}

1. https://www.public-montessori.fr/

2. 55 établissements font partie du réseau Don Bosco et plus d'une centaine pour le réseau lasallien en France. https://www.lasallefrance.fr/wp-content/uploads/2016/04/ doc_pel_2012_54.pdf

3. Ce petit "lexique " est repris par l'ensemble des sites qui diffusent les idées de l'école à domicile, qu'il s'agisse du mouvement Colibris ou du site du festival de l'école pour la vie. https://printemps-education.org/actualites/non-classe/petit-lexique-des-pedagogies-

differentes/.

4. Ibid.

5. https://www.festival-ecole-de-la-vie.fr/

6. https://www.colibris-lemouvement.org/passer-a-laction/creer-son-projet/creer-un-lieudeducation-alternatif

7. https://www.ecole-domaine-du-possible.fr/

8. http://biennale-education.org/

9. http://www.steiner-waldorf.org; http://www.montessori-france.asso.fr; http://www.icempedagogie-freinet.org.

10.

https://www.cemea.asso.fr/IMG/pdf/

plaquette_presentation_biennale_educ_nouvelle2017_version_sept2016.pdf

11. http://www.festival-ecole-de-la-vie.fr/principaux-courants-alternatifs/

12. https://www.public-montessori.fr/

13. https://aquitaine.sgen-cfdt.fr/actu/formation-syndicale-la-pedagogie-montessori/ ; http://82.snuipp.fr/spip.php?article1410

14. https://www.celinealvarez.org/ . https://www.celinealvarez.org/notre-demarche

15. https://www.cecp.be/circulaire/circulaire-6696-projet-daccompagnement-dequipesdimplantations-maternelles-par-celine-alvarez-appel-a-projets-2018-2019/

16. Les écoles démocratiques sciences humaines 2018

17. Synthèse des travaux du Conseil National de l'Innovation pour la Réussite Educative 2013-2014 [en ligne], http://cache.media.education.gouv.fr/ file/11_Novembre/91/4/2014_rapport_cnire_web_366914.pdf

18. http://www.crdp-lyon.fr/podcast/conference-peut-on-repenser-l-education-a-la-lumieredes-recherches-recentes-en-neurosciences-affectives-conference

19. https://www.filliozat.net/

20. https://www.ateliers-filliozat.com/decouverte/

21. https://www.festival-ecole-de-la-vie.fr/neurosciences-et-education-positive/

22. https://primabord.eduscol.education.fr/qu-est-ce-que-les-neurosciences-cognitives

23. Ibid.

24. https://printemps-education.org/actualites/non-classe/petit-lexique-des-pedagogiesdifferentes/. Ce petit «lexique» est repris par l'ensemble des sites qui diffusent les idées de l'école à domicile, qu'il s'agisse du mouvement Colibris ou du site du festival de l'école pour la vie.

25. http://www.lesenfantsdabord.org; http://laia.asso.free.fr; http://cise.fr ; http:// parentsinstructeursdefrance.blogspot.be ; http://association-unie.blogspot.be ; https:// lecoleestlamaison.blogspot.be/

26. http://www.linstantpresent.eu/fr/ ; http://ecole-vivante.com/

27. https://printemps-education.org/

28. http://www.festival-ecole-de-la-vie.fr/ 
29. https://www.colibris-lemouvement.org/passer-a-laction/creer-son-projet/creer-un-lieudeducation-alternatif ; http://www.festival-ecole-de-la-vie.fr/comment-creer-une-ecolealternative/

30. Ibid

31. Ibid

32. http://www.villagedepourgues.coop/immersion-avec-ramin/ http://creer-son-ecole.com/

\section{RÉSUMÉS}

Comment définir ces pédagogies alternatives et comprendre leurs finalités éducatives? Nous avons choisi de mettre en avant les principes fédérateurs de ces pédagogies sans toutefois omettre les oppositions et les conflits entre ces mouvements. Quels sont les invariants théoriques, les pratiques et les finalités communes de ces écoles et de ces " pédagogies " ? Quelles sont leurs aspirations, leur volonté de changer le paradigme scolaire? Ces écoles sont-elles, comme la plupart des acteurs de ces écoles le soulignent, l'illustration de l'essor d'une autre vision de notre société avec des rapports humains plus bienveillants et plus fraternels pour le bien-être des enfants ou, de façon non assumée, une volonté de repli sur soi ? Une cartographie nous est apparue nécessaire pour expliciter la variété de la nébuleuse des courants dits des pédagogies alternatives mais aussi pour analyser leurs caractéristiques.

How to define these alternative pedagogies and understand their educational goals? We have chosen to put forward the unifying principles of these pedagogies without omitting the oppositions and the conflicts between these movements. What are the theoretical invariants, practices and common goals of these schools and "pedagogies"? What are their aspirations, their willingness to change the school paradigm? Are these schools the illustration of the development of another vision of our society with more fraternal human relationships for the well-being of children, or a will to withdraw into oneself? A cartography appeared to us necessary to explain the variety of the nebula of alternative pedagogies but also to analyze their characteristics.

\section{INDEX}

Mots-clés : pédagogies alternatives, éducation nouvelle, neurosciences, développement personnel, parents

Keywords : Alternative Pedagogies, New Education, Neuroscience, Personal Development, Parents

\section{AUTEUR}

\section{SYLVAIN WAGNON}

Lirdef, Faculté d'Education, Université de Montpellier 